\title{
Human Toxocariasis Presenting with Fever and Colestatic Hepatitis: An Underestimated but Current Zoonosis
}

\author{
Vera Sicbaldi $^{{ }^{*}}$, Andrea Bellodi ${ }^{1}$, Elisa Molinari ${ }^{1}$, Valerio Del Bono ${ }^{2}$, Eleonora Arboscello ${ }^{1}$, \\ Riccardo Ghio ${ }^{1}$ \\ ${ }^{1}$ Clinic of Internal Medicine 3, IRCSS San Martino IST, Genoa, Italy; ${ }^{2}$ Clinic of Infectious Disease, IRCSS San Martino IST, Genoa, \\ Italy. \\ Email: *verasic@libero.it
}

Received September $8^{\text {th }}, 2012$; revised October $20^{\text {th }}, 2012$; accepted November $20^{\text {th }}, 2012$

\begin{abstract}
Human toxocariasis is a widespread parasitic disease caused by ingestion of Toxocara canis or catis larvae or eggs. Parasitic diseases are uncommon in industrialized countries, yet this problem has not disappeared. Parasitic diseases can cause different syndrome (visceral larva migrans, ocular larva migrans) and the clinical features can be confusing. Severe organ involvement affecting the liver, lungs, nervous central system and eyes can lead to serious damage. We present a case of toxocariasis presenting with fever, desaturation and cholestatic hepatitis. Only the subsequent appearance of eosinophilia helped us to arrive at the correct diagnosis. Prompt diagnosis allowed specific therapy avoiding permanent complications.
\end{abstract}

Keywords: Toxocariasis; Visceral Larva Migrans; Ocular Larva Migrans; Parasitosis; Cholestatic Hepatitis; Eosinophilia

\section{Introduction}

Toxocariasis is secondary to a human infection caused by larvae of Toxocara canis (T. canis) or Toxocara catis ( $T$. catis). The definitive hosts are represented by pets in whom helmints live as adult stage in the lumen of the small intestine. The human infection is acquired through the direct ingestion of nematode or its eggs or transplacentally. Human infestation is common, mainly among children because of contact with contaminated soil or infected puppies. Another possible way of infection, especially in adults, is comsumption of raw meat from infected animals. Humans are infected with $T$. canis by swallowing invasive eggs which release larvae in the upper small bowel. Larvae can penetrate the bowel's wall gaining access to portal venous circulation and then invading hepatic tissue leading to two main different clinical syndromes such as visceral larva migrans (VLM) and ocular larva migrans (OLM) [1]. Prevalence of parasitosis is different from country to country but it is in constantly increase in urban areas [2]. Even if it is underestimated, recent epidemiological data demonstrate the widespread prevalence of Toxocara human infection. Infact it appears to probably be the most prevalent helminthiasis in industrialized countries, representing a typical

*Corresponding author. neglected and underestimated human health problem [3].

\section{Case Report}

A 66-year-old immunocompetent man was admitted to our Department because of fever lasting from about a week associated with nausea and vomiting that did not respond of antibiotic therapy with levofloxacin. The patient was a florist. He made a trip to Libya few months earlier and the night before the onset of symptoms he had mushrooms and cooked fish for dinner. In his medical history there were benign prostatic hyperplasia, surgical excision of bladder papillary urothelial low grade carcinoma, extracorporeal lithotripsy for uroteral lithiasis. At the physical examination only a mild liver enlargement was noticed; there was a little decrease in $\mathrm{SpO}_{2}(94 \%$ in room air) with no signs of dyspnoea. Laboratory tests showed mild normocytic anemia, an evident increase of inflammatory markers (CRP 85 mg/dl; ESR 114 ml; serum fibrinogen $687 \mathrm{mg} / \mathrm{dl}$ ), cholestatic hepatitis with AST/ALT ratio inversion. There was also increase of ferritin and both IgA and IgE. All serologic tests for hepatitis viruses (HAV, HBV, HCV, HEV, EBV and CMV) were negative. The search for autoantibodies (ANA, ANCA, AMA, ASMA, LKM) showed a non-specific pattern and a non-significant antibody titer. All the cultural microbiological tests (blood, urine and stool) were 
negative. Two iodine stool microscopic examination carried out in different days turned out to be negatives for parasitic larvae or eggs. Chest X-ray did not show any parenchimal involvement. An abdominal ultrasonography did show mild enlargement of the liver without any ultrasonographic abnormality. The biliary tree was normal. A week after the admission the blood count showed eosinophilic leukocytosis $\left(6.7 \times 10^{9} / \mathrm{L}\right)$. At that point other diagnostic tests were performed: serolocic tests for Ameba, Schistosoma, Echinococco, Giardia and Toxocara. A chest CT scan showed bilateral interstitial "ground glass" infiltrates (Figure 1) and hypodense cystic areas in the liver. Meanwhile serological tests showed the presence of the anti-toxocara antibodies. Those, the diagnosis of toxocariasis (visceral larva migrans, VLM) was made on the bases of positive serological results, cholestatic hepatitis and eosinophilic leukocytosis. Ophthalmoscopic examination was then performed showing no fundus injuries. Treatment with albendazole $(400 \mathrm{mg}$ twice/day for 5 days) in combination with corticosteroid (prednisone $25 \mathrm{mg}$ once/day) was started. A three weeks follow up showed complete normalization of blood tests (blood count, IgE and liver enzymes).

\section{Discussion}

The most frequent clinical manifestation of toxocariasis are VLM and OLM; clinical features are nonspecific symptoms such as fever, weakness, pruritus, dyspnoea, anorexia, headache, abdominal pain, nausea, vomiting, drowsiness [4]. Human toxocariasis also has been associated with various allergy-releated syndromes including chronic urticaria, skin rash, reactive arthritis, bronchial asthma, vasculitis and or angioedema $[5,6]$.

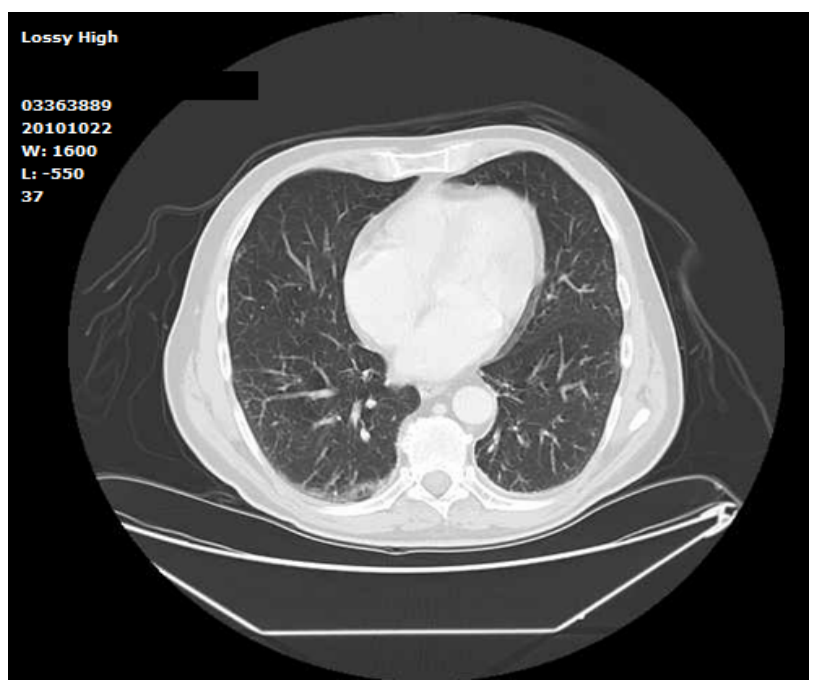

Figure 1. Bilateral lung "ground-glass" infiltrations which determined desaturation and mild dyspnoea.

\subsection{Visceral Larva Migrans}

Visceral larva migrans is caused by the migration of larvae through the internal organs of humans and the resulting inflammatory reaction. Multiple organs and tissues may be affected, especially the liver and the lungs. Toxic products are released with the onset of a local eosinophilic granulomatous response. Examination may reveal hepatomegaly, lymphadenitis and sometimes pleural effusions [2]. Severe cases can lead to myocarditis or respiratory failure $[7,8]$. Involvement of the central nervous system is infrequent but dangerous and lifethreatening. It can occur with seizures or motor deficits [9]. Growth of liver multiple granulomas with necrotic and/or fibrotic areas could lead to acute cholestatic hepatitis [1]. Our patient presented clinical features suggesting cholestatic hepatitis in absence of radiological findings of biliary lithiasis, history of alcoholic or drugs abuse. Auto antibodies were negative and serological test for the most common hepatotropic viruses were also negative. Intoxication seemed to be less probable, so more diagnostic tests were necessary. Desaturation and fever suggested wider systemic involvement. CT scan associated to the anti toxocara antibodies strenghtened the hypothesis of parasitic infestation.

\subsection{Ocular Larva Migrans}

Ocular larva migrans is caused by migration of larva into the posterior segment of the eye and tends to occur in older children and young adults. Patients may present with decreased vision, red eye and leukokoria (white appearance of the pupil) [10]. In the retina, especially at the macula, can be observed granulomas, chorioretinitis, uveitis and papillitis. Inflammatory masses called snow banks may appear in the peripheral vitreous. Unilateral visual loss, retinal fibrosis, retinoblastoma and retinal detachment can be other possible features of the ocular disease. Eye damage usually become apparent after months or years from infection, including subclinical infection [2]. Our patient did not show any ocular symptom, but fundus examination was though performed in order to exclude a potentially severe involvement.

\subsection{Diagnosis and Treatment}

The diagnosis of toxocariasis is based on clinical findings in association with laboratory data. Eosinophilia is a characteristic feature, but it can also be found leukocytosis and hypergammaglobulinemia. Confirmation of diagnosis is based on the antibodies to Toxocara detected by a serologic test (enzyme-linked immunosorbent assay, ELISA) [11]. Furthermore, increased levels of serum IgE may be present. Sometimes, instrumental examination like as chest radiography, ultrasonography and CT scan 
are useful to confirm diagnosis [4]. In previous reports are described many cases without eosinophilia [1]. In our experience eosinophilia and laboratory cholestatic signs together with chest-CT scan were decisive to guide toward parasitosis. These clues were supported by positivity of anti-toxocara antibodies which confirmed the diagnosis. The most difficult cases may require the execution of hepatic biopsy to confirm the presence of Toxocara larvae. However it is rarely performed. A careful opthalmoscopic examination must be performed in all cases of known or suspected toxocariasis [12]. While less severe forms require no treatment, in patients with severe involvement of lung, heart, central nervous system or liver corticosteroids should be prescribed (for example prednisone $1 \mathrm{mg} / \mathrm{kg} /$ day orally). Although several anthelmintics medications had been tested in animal models, only a few drugs are available for the treatment of the human disease due to the lack of randomized studies in humans. Thiabendazole, mebendazole, albendazole and diethylcarbamazine are the drugs most commonly used [2]. In ocular involvement anthelmintic therapy is completely ineffective, therefore corticosteroid treatment (either topical or systemic) is suggested. Corticosteroids reduce local inflammation caused by toxic products of Toxocara and improve symptoms quickly. They are helpful to avoid ocular permanent damages. Ocular laser therapies are also described [4].

\section{Conclusion}

Our patient was an immunocompetent host but he acquired the disease, probably due to his predisposing job (handling potentially contaminated soil). Our experience highlight the importance of know parasitic infestations and their pathogenetic ways, even in industrialized countries. In fact they can mimic symptoms of several other systemic diseases, making hard their recognition. Non specific presentation symptoms associated to poor awareness of this problem can lead to under diagnosis. Thinking also of Toxocara and others infestation in hepatic and lungs injury, with or without eosinophilia, can make sometimes the diagnostic path easier. This can lead to a faster recognition and prompt treatment avoiding permanent organ damage. Simple hygienic measures can be adopted to prevent infestations such as avoiding casual stool ingestion, expecially in children. The main risk factors identified are domestical animals (cats and dogs, expecially puppies). Treatment of pets with benzimida- zoles should be adopted as preventing measure to avoid human infestation. Periodical animals stool examination can also be suggested.

\section{REFERENCES}

[1] N. Leone, et al., "Hepatic Involvement in Larva Migrans of Toxocara canis: Report of a Case with Pathological and Radiological Findings," Digestive and Liver Disease, Vol. 38, No. 7, 2006, pp. 511-514. doi:10.1016/j.dld.2005.07.007

[2] D. Despommier, "Toxocariasis: Clinical Aspects, Epidemiology, Medical Ecology and Molecular Aspects,” Clinical Microbiology Reviews, Vol. 16, No. 2, 2003, pp. 265-272.

[3] J. Chen, et al., "Advances in Molecular Identification, Taxonomy, Genetic Variation and Diagnosis of Toxocara spp.," Infection, Genetics and Evolution, Vol. 12, No. 7, 2012, pp. 1344-1348. doi:10.1016/j.meegid.2012.04.019

[4] J.-F. Magnaval, et al., "Highlights of Human Toxocariasis," The Korean Journal of Parasitology, Vol. 39, No. 1, 2001, pp. 1-11.

[5] A.-P. Bellanger, et al., "Rheumatoid Purpura Associated with Toxocariasis," Canadian Family Physician, Vol. 57, No. 12, 2011, pp. 1413-1414.

[6] L. Lopez Mde, “Toxocara canis and Bronchial Asthma," Medicina Buenos Aires, Vol. 70, No. 1, 2010, pp. 75-78.

[7] K. Enko, et al., "Fulminant Eosinophilic Myocarditis Associated with Visceral Larva Migrans Caused by Toxocara canis Infection,” Circulation Journal, Vol. 73, No. 7, 2009, pp. 1344-1348. doi:10.1253/circj.CJ-08-0334

[8] A. K. Bartelink, "Acute Respiratory Failure Due to Toxocara Infection,” Lancet, Vol. 343, No. 8881, 1993, p. 1234.

[9] B. Hoffmeister, et al., "Cerebral Toxocariasis after Consumption of Raw Duck Liver," The American Journal of Tropical Medicine and Hygiene, Vol. 76, No. 3, 2007, pp. 600-602.

[10] O. Verallo, et al., "Diagnostic Aspects and Retinal Imaging in Ocular Toxocariasis: A Case Report from Italy," Case Reports in Medicine, Vol. 2012, 2012, Article ID: 984512.

[11] M.-V. Bojanich, et al., "An Evaluation of the Dot-Elisa Procedure as a Diagnostic Test in an Area with a High Prevalence of Human Toxocara canis Infection,” Memorias do Instituto Oswaldo Cruz, Rio de Janeiro, Vol. 107, No. 2, 2012, pp. 194-197.

[12] G. Rubinsky-Elefant, "Human Toxocariasis: Diagnosis, Worldwide Seroprevalences and Clinical Expression of the Systemic and Ocular Forms," Annals of Tropical Medicine and Parasitology, Vol. 104, No. 1, 2010, pp. 3-23. 